\title{
Activated carbon derived from human hair as electrode material for supercapacitors
}

\author{
Robin Rathaur ${ }^{1}$ and Kamal K. Kar ${ }^{1,2^{*}}$ \\ ${ }^{1}$ Advanced Nanoengineering Materials laboratory, Materials Science Programme \\ ${ }^{2}$ Advanced Nanoengineering Materials laboratory, Department of Mechanical Engineering, \\ Indian Institute of Technology Kanpur, Kanpur-208016, India. \\ *Email: kamalkk@iitk.ac.in
}

Due to increasing global energy crisis and environmental problems, it is important to develop novel technologies for clean and sustainable energy. Carbon-based materials, especially nanostructured porous carbons have been demonstrated to be attractive for clean energy applications. In view of this, supercapacitors are of prime interest and are able to store energy in the form of electrical charge. They attract the attention due to high power density, short charging time and long cycling life. Human hair, an accessible waste, consists of $\sim 52 \%$ carbon, $17 \%$ nitrogen, $22 \%$ oxygen, $6 \%$ hydrogen, $5 \%$ sulfur and small amounts of magnesium, arsenic and other materials. Activated carbon derived from human hair is another way to generate electrode material for supercapacitors $[1,2]$. In this study, the focus is on synthesis of heteroatom-doped carbon material via carbonization of human hair. Porous carbon material with high surface area induced by $\mathrm{KOH}$ activation during carbonization has been formed.

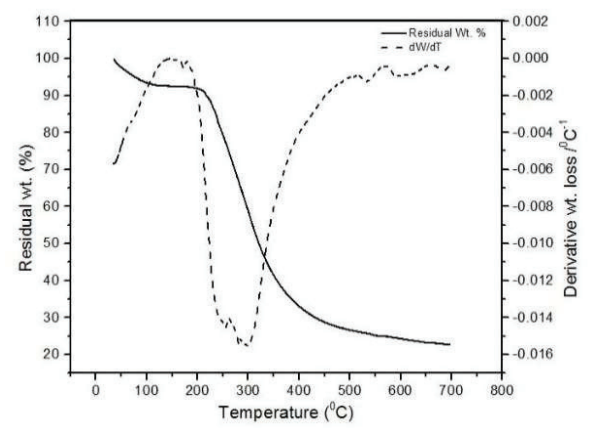

Figure 1: TGA of human hair showing residual weight $(\%)$ and temperature derivative of weight loss $(\mathrm{dW} / \mathrm{dT})$ curve

First human hair samples were taken and carbonized at the temperature $300,500,700$ and $900^{\circ} \mathrm{C}$. Thermal behavior of the human hair has been studied by thermogravimetric analysis (TGA) (Figure 1) and also structural characteristics such as crystallinity, surface morphology, surface area analysis has been done followed by electrochemical characterizations such as cyclic voltammetry (CV) (Figure 2).

The results show that the human hair derived activated carbons have powerful potential toward their application as electrode material for supercapacitors.

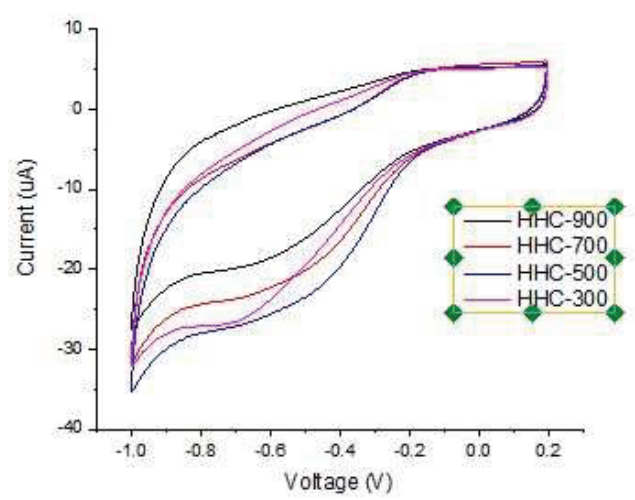

Figure 2: Cyclic voltammogram of human hair derived activated carbons in $0.1 \mathrm{M} \mathrm{KOH}$ at a scan rate of $50 \mathrm{mV} / \mathrm{s}$

\section{References}

1. Wenjing Qian,a Fengxia Sun,b Yanhui Xu,b Lihua Qiu,a Changhai Liu,c Suidong Wang, Energy Environ. Sci., 2014, 7, 379

2. Xiaojun Liu,a Weijia Zhou,*a Linjing Yang,a Ligui Li J. Mater. Chem. A, 2015, 3, 8840 